\title{
The astrometric accuracy of "Carte du Ciel" plates and proper motions in the field of the open cluster NGC $1647^{\star}$
}

\author{
M. Geffert ${ }^{1,3}$, P. Bonnefond ${ }^{2}$, G. Maintz ${ }^{1}$ and J. Guibert ${ }^{2}$ \\ 1 Sternwarte der Universität Bonn, Auf dem Hügel 71, D-53121 Bonn, Germany \\ 2 Centre d' Analyse des Images, Bâtiment Perrault, Observatoire de Paris, 77 avenue Denfert Rochereau, F-75014 \\ Paris, France \\ 3 Observatoire de Bordeaux, BP. 89, F-33270 Floirac, France
}

Received September 4, 1995; accepted January 19, 1996

\begin{abstract}
The astrometric accuracy of triple image Carte du Ciel plates has been analysed using plates of the open cluster NGC 1647. A new multithreshold technique was used for the treatment of the triple image plates. Accuracies ranging from 100 mas to 200 mas were found. We determined proper motions with a median accuracy of $1.6 \mathrm{mas} / \mathrm{a}$ for 2220 stars in the field of NGC 1647. The membership probabilities of stars in the cluster region agree very well with the data of Francic (1989). Due to a significant difference of the proper motions of SZ Tau and the cluster members, SZ Tau seems not to be a member of NGC 1647.
\end{abstract}

Key words: astrometry — stars: individual: SZ Tau — Galaxy: open clusters: individual: NGC 1647

\section{Introduction}

The forthcoming HIPPARCOS proper motion system will, due to its accuracy and homogeneity, lead to a large progress for many kinematical studies. Nevertheless, for some purposes the number of stars within a certain area of the sky as well as the limiting magnitude are still too small. Therefore it seems desirable to extend the HIPPARCOS proper motions to a complete sample of stars with fainter magnitudes. Such an extension may be done with earthbound techniques, if first epoch plates of the interesting region are available.

The plates of the Carte du Ciel project, (which was started in 1887) form, with respect to their age and their nearly complete coverage of the whole sky, material well suited for the determination of proper motions. Since the observations were conducted at several observatories, each observatory having a dedicated declination zone, the plate material is distributed over the whole world.

There were two kinds of observations for the Carte du Ciel project: (1) the catalogue plates, which were measured completely and whose positions have been published in the Astrographic Catalogue; (2) the deeper plates $(V=14)$ for the Carte du Ciel atlas, which have been mea-

Send offprint requests to: M. Geffert, Bonn

${ }^{\star}$ Table 7 is only available in electronic form at the Centre des Donées Strasbourg (CDS) via anonymous ftp 130.79.128.5; see the Editorial in A\&A 280, E1 (1993) sured only in individual cases. In most zones these plates are the result of equal exposures with a positional offset such that the stars form a triangle. For the brighter stars, the images on these plates are blended, which makes astrometric use of this material very difficult. In the following, we will denote plates exposed in that way as "triple image plates".

While earlier attempts (Brosche \& Geffert 1988; Brosche et al. 1989) have given first indications about the astrometric accuracies of single exposed Carte du Ciel plates, Lattanzi et al. (1991) used positions from triple images of Carte du Ciel plates without special treatment. Recent investigations (Dick et al. 1993 and references herein, Ortiz-Gil et al. 1995) try to develop special fitting algorithms for the triple image plates of the Carte du Ciel. In this paper we have made a proper motion study of the open cluster NGC 1647 using a a multithreshold procedure for the determination of $x$ and $y$ positions of triple image Carte du Ciel plates (Bonnefond 1991). NGC 1647 is well suited for testing the accuracy of Carte du Ciel plates, since it is located at the border of four Carte du Ciel plates (two with triple images), which completely overlap the cluster. We expect therefore, especially by the comparison with independent catalogues, information about the astrometric accuracy at the border of the plates. A possible astrometric use of larger fields covered by Carte du Ciel plates needs positions from the 
Table 1. The observational data used in this work

\begin{tabular}{lrrrrr}
\hline Telescope & $\begin{array}{r}\text { Scale } \\
{\left[{ }^{\prime \prime} / \mathrm{mm}\right]}\end{array}$ & Epoch & Field & $V_{\text {lim }}$ & $N$ \\
\hline Double refractor of Bonn, now located at Hoher List & 40 & 1988 & $1.6^{\circ} \times 1.6^{\circ}$ & 15.0 & 3 \\
AG Astrograph from Bonn & 100 & $1932-1933$ & $5^{\circ} \times 5^{\circ}$ & 12.5 & 3 \\
AG Astrograph from Bonn & 100 & $1981-1982$ & $5^{\circ} \times 5^{\circ}$ & 11.5 & 7 \\
Schmidt telescope of CERGA & 66 & 1989 & $5^{\circ} \times 5^{\circ}$ & 16.0 & 2 \\
Carte du Ciel telescope of Observatoire de Paris & 60 & $1897-1924$ & $2^{\circ} \times 2^{\circ}$ & $9-14.5$ & 10 \\
\hline
\end{tabular}

$N$ is the number of exposures

border of the plates, since the overlap of the Carte du Ciel plates very often is equal or less than $10^{\prime}$.

\section{Observations and measurement}

The base of this investigation are four plates taken with the Carte du Ciel astrograph of Paris taken between 1897 and 1924. Two of these plates were taken for the Catalogue program having three exposures with limiting magnitudes of $V=11.5$ and fainter. The angular distance between the exposures is about $13^{\prime \prime}$ which, together with the short exposure times, seems to be large enough so that the positions of the images were not disturbed by the corresponding images of the other exposures. The two plates overlap in right ascension to a degree of $15^{\prime}$, while NGC 1647 is located in the centre of the overlap region respectively at the border of each of the two plates. For our work, we have used only the two deeper exposures of each plate. The other two plates overlap in declination in the same way, which means that the cluster is located at the northern border of one plate and at the southern border of the other plate. Each of these plates contains three nearly equal exposed images, which are separated by about $12^{\prime \prime}$, which led to blended images for the bright stars.

In addition to the Carte du Ciel plates mentioned above, we used plates taken with the $(D=0.7 \mathrm{~m}, f=3$ m) Schmidt telescope of CERGA, the $(D=0.3 \mathrm{~m}, f=5$ $\mathrm{m})$ double refractor of Bonn and the $(D=0.2 \mathrm{~m}, f=2 \mathrm{~m})$ AG astrograph of Bonn. The AG astrograph was moved in 1973 to the new building of the Astronomischen Institute within Bonn, where the second epoch plates were taken. The complete observational material is listed in Table 1. All plates were digitised with the MAMA (Machine Automatique à Mesurer pour l' Astronomie) in Paris (Guibert et al. 1990; Berger et al. 1991), which is developed and operated by INSU/CNRS. Only two refractor plates had been scanned at the PDS 2020GM ${ }^{\text {plus }}$ in Münster. The procedure of the determination of the $x$ and $y$ positions for the triple image plates is described in the next chapter, while for the other plates the standard routines of MAMA and the PDS were used.
Table 2. Internal accuracy of Carte du Ciel plates determined from comparison of different exposures on the same plate. For the triple image plates 2970 and 3219 different methods of determination of positions have been applied (see Sect. 3)

\begin{tabular}{lrrrrr}
\hline NP & Triple image & $\begin{array}{r}\sigma_{\alpha} \\
{[\mathrm{mas}]}\end{array}$ & $\begin{array}{r}\sigma_{\delta} \\
{[\mathrm{mas}]}\end{array}$ & NS & Method \\
\hline 1394 & no & 80 & 80 & 58 & \\
1513 & no & 140 & 150 & 45 & \\
2970 & yes & 130 & 110 & 530 & $\mathrm{~A}$ \\
2970 & yes & 120 & 100 & 530 & $\mathrm{~B}$ \\
3219 & yes & 160 & 190 & 511 & $\mathrm{~A}$ \\
3219 & yes & 150 & 160 & 511 & $\mathrm{~B}$ \\
\hline
\end{tabular}

$\mathrm{NP}$ is the plate number

NS is the number of stars

\section{The treatment of the triple images}

For the determinations of the positions of the triple images on the Carte du Ciel plates a special procedure was applied. Each plate was digitised and, for nine different density thresholds, all detected objects and their positions were stored. The detection of stars on the old plates was hampered by the Reseau lines and scratches on the old emulsions. However, since scratches and Reseau lines normally have larger ratios of the principal axes of the images and smaller ratios of the image area with respect to the area of the enclosing box, most of these artifical objects could be removed from the data by setting constraints on these ratios. The final positions were determined by the centroid of the detected pixels, weighted by their density. The next step was the automatic reorganisation of all positions for the different thresholds and the three exposures of one star. In a further step the position of each image of each of the three exposures was calculated separately in two different ways. Method A uses only the position from the highest threshold on which the object was detected. Method B averages the positions of all thresholds in which the object was found. A complete description of the procedures is given in Bonnefond (1991). Both 
methods give positions of nearly all stars on the plate within the magnitude interval from $V=9.5$ up to the plate limit of $V=14.5$. Images of the brighter stars blend so much, that no positions could be derived with our software.

\section{The comparison of rectangular coordinates from different exposures of one plate}

In order to determine the astrometric accuracy of Carte $\mathrm{du}$ Ciel plates, we have in a first step transformed by an affine transformation the positions of different exposures on the same plate to one rectangular system and determined mean position and $\mathrm{rms}$ of the deviations for each exposure. Under the assumption that all exposures contribute in the same way to the deviations, we may calculate from the rms mentioned above the contribution of one single exposure. In the following we will denote this as the internal accuracy of the Carte du Ciel plates. The results of our four Carte du Ciel plates are presented in Table 2. A large number of images on the plate are likely disturbed by the reseau-lines on the plate, by blended images, or by scratches and other contaminations of the plates. Therefore we have excluded from the data set all stars with deviations larger than $3 \sigma$ in order to get a homogenious data set for the plates.

The data in Table 2 show that the different methods of getting the positions from the images agree quite well. The mean positions of all thresholds are slightly more accurate than the positions from the first threshold on which the object was detected. The more suprising result is that both plates have different internal accuracies. In order to look for systematic patterns of the differences and especially for a possible Kostinsky effect for triple image plates as found in Dick et al. (1993), we have plotted separations of the images of the two exposures with respect to the peak intensity of the stars and with respect to the distances of the stars from the centre of the plate. These are shown for two exposures of the plate 2970 in Figs. 1 and 2 . In general, the diagrams for both methods of calculating the positions from the triple image plates (see Sect. 3 ) are nearly identical. We did not find a Kostinsky effect on the plates as was found earlier by Dick et al. (1993). Most diagrams showed a small or neglegible trend in the distances with repect to internal magnitude or distance from the plate centre (as indicated in Figs. 1 and 2). Only stars at larger distances $(>60 \mathrm{~mm})$ from the plate centre show in some cases a tendency of larger but also smaller separations between the two exposures.

With these investigations we could only detect differences between two exposures of the same plate. It is a well known fact that the Carte du Ciel images show optical errors in particular at the border of the plates. If systematic astrometric errors would result from these optical errors we could not detect them by comparison of different ex-

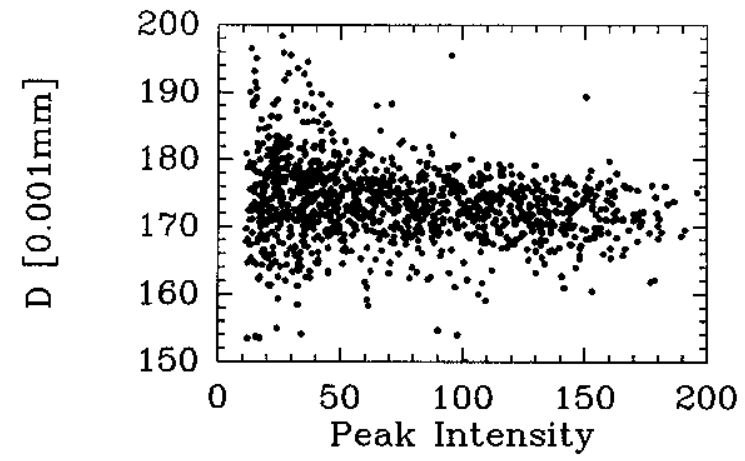

Fig. 1. Separation of two images of the same star on two exposures of the triple image plate 2970 with respect to internal peak intensity of the stars. Note that the brightest stars have $V=9.5$ and the faintest ones $V=14.5$

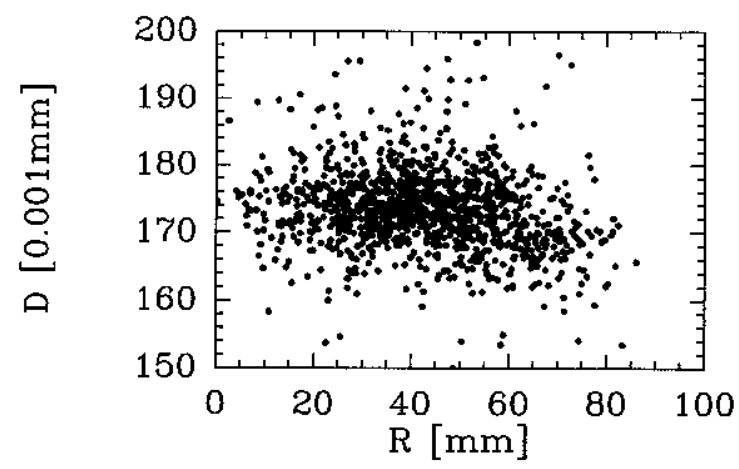

Fig. 2. Separation of two images of the same star on two exposures of the triple image plate 2970 with respect to the distance of the star from the plate centre

posures of one plate, since they would affect all exposures in the same way.

\section{Comparison with independent catalogues}

For an estimation of the systematic errors of the Carte du Ciel plates, especially at the border of the plates, we have reduced the Carte du Ciel plates with independent astrometric catalogues. The first catalogue was the catalogue of 122 stars with positions and proper motions derived from measurements of the exposures given in Table 1 . We have limited our investigation to a field of $1.6^{\circ} \times 1.6^{\circ}$ around the cluster centre (the size of the refractor plates). An central overlap algorithm was used for the determination of the positions and proper motions. As input catalogue we used 34 PPM stars. Since Geffert et al. (1995) have shown that a direct reduction to the PPM catalogue could cause larger systematic errors, we have determined a catalogue of positions for the epoch 1950 for all stars, and then started the reduction with stars having small proper motions in the same way as described in Geffert et al. (1995). 
Table 3. Rms of the deviations of measurements of Carte du Ciel exposures from our preliminary catalogue. Note that for the reduction of the exposures of plate 3219 Coma terms had to be included to the standard reduction using terms with polynomials up to second order of the rectangular coordinates $x$ and $y$

\begin{tabular}{lrrrrr}
\hline NP & Triple image & $\begin{array}{r}\sigma_{\alpha} \\
{[\mathrm{mas}]}\end{array}$ & $\begin{array}{r}\sigma_{\delta} \\
{[\mathrm{mas}]}\end{array}$ & NS & Method \\
\hline 1394 & no & 150 & 150 & 44 & \\
1513 & no & 150 & 150 & 39 & \\
2970 & yes & 150 & 140 & 55 & $\mathrm{~A}$ \\
2970 & yes & 140 & 140 & 55 & $\mathrm{~B}$ \\
3219 & yes & 200 & 200 & 54 & $\mathrm{~A}$ \\
3219 & yes & 200 & 190 & 54 & $\mathrm{~B}$ \\
\hline
\end{tabular}

$\mathrm{NP}$ is the plate number

NS is the number of stars

2970 and 3219 are the triple image plates

Table 4. Rms of the deviations of measurements of Carte du Ciel exposures from the catalogue of Francic (1989). Note that for the reduction of the exposures of plate 3219 Coma terms had to be included to the standard reduction using terms with polynomials up to second order of the rectangular coordinates $x$ and $y$

\begin{tabular}{lrrrrr}
\hline NP & Triple image & $\begin{array}{r}\sigma_{\alpha} \\
{[\mathrm{mas}]}\end{array}$ & $\begin{array}{r}\sigma_{\delta} \\
{[\mathrm{mas}]}\end{array}$ & NS & Method \\
\hline 1394 & no & 110 & 80 & 36 & \\
1513 & no & 130 & 100 & 39 & \\
2970 & yes & 160 & 160 & 128 & $\mathrm{~A}$ \\
2970 & yes & 180 & 180 & 128 & $\mathrm{~B}$ \\
3219 & yes & 140 & 180 & 89 & $\mathrm{~A}$ \\
3219 & yes & 170 & 190 & 89 & $\mathrm{~B}$ \\
\hline
\end{tabular}

$\mathrm{NP}$ is the plate number

NS is the number of stars

2970 and 3219 are the triple image plates to be included in the reduction. Table 3 gives the mean deviations for each plate.

In a second step we also compared the positions measured on the Carte du Ciel plates with the catalogue of Francic (1989). Unfortunately he gives in his catalogue only the data of probable cluster members. The rectangular positions were transformed into right ascension and declination with the help of our catalogue. Since the rectangular positions of Francic (1989) were given only in hundreths of $\mathrm{mm}$, the spherical positions have an accuracy no better than 60 mas. The results of the comparison with this catalogue is given in Table 4 . Since the errors of our catalogues are below the positional errors of our measurements of the Carte du Ciel plates, the Tables 3 and 4 reflect the astrometric accuracy of our Carte du Ciel plates. Note that the comparison with the catalogue of Francic (1989) is based on a smaller field, but down to fainter magnitudes than the comparison with our catalogue, which explains the differences of our astrometric accuracy in Table 3 and 4 . Unfortunately in both cases the number of comparison stars is not very high. Again we see a significant difference in the astrometric quality of the two Carte $\mathrm{du}$ Ciel plates which is also manifested in the existence of Coma terms in only one plate. Nevertheless, even the astrometric accuracy of about 200 mas for the astrometric accuracy of plate 3219 is remarkable, bearing in mind that we are dealing with positions from the extreme border of the plate. Because of its smaller deviations from the catalogue, we have for the following chosen the positions of the triple image plates, which were determined from the first significant threshold (Method A).

Table 5. Mean proper motion differences and rms between our final catalogue and the PPM catalogue (PPM) and the catalogue of Francic (1989) (FRAN)

\begin{tabular}{lrrr}
\hline Catalogue & $\begin{array}{r}\Delta_{\mu_{\alpha} \cos \delta} \\
{[\mathrm{mas} / \mathrm{a}]}\end{array}$ & $\begin{array}{r}\Delta_{\mu_{\delta}} \\
{[\mathrm{mas} / \mathrm{a}]}\end{array}$ & $\mathrm{NS}$ \\
& & \\
\hline PPM & $+3.7 \pm 4.8$ & $+0.9 \pm 4.7$ & 34 \\
FRAN & $-0.5 \pm 1.5$ & $+1.1 \pm 2.4$ & 165 \\
\hline
\end{tabular}

NS is the number of stars used for comparison.

The measurements of our Carte du Ciel exposures were then reduced with this catalogue and the rms of the deviations in right ascension and declination were determined. Since the limiting magnitude of most of our old plates (except the Carte du Ciel plates) was only 12, and since the Carte du Ciel plates cover each only half of the cluster area, the comparison is based on only 50 to 60 stars. Since the positional accuracy of our preliminary catalogue for the epoch of the carte du Ciel plates is about $40-$ 50 mas, the deviations will be mainly due to the uncertainty of the Carte du Ciel positions. For the reduction of the plate 3219 coma terms were significant and had

\section{The final catalogue}

The complete observational material of Table 1 was used for the determination of the final catalogue. The exposures of the triple image plates were taken in the reduction as independent exposures. As described in Sect. 5, the final catalogue in the first step of the reduction was calculated by using only the positions of all those stars having small proper motions. Their positions were determined with respect to the PPM catalogue for the mean epoch of the observations. For the following steps a central overlap 
Table 6. The parameters of the bivariate Gaussian fit for the determination of membership probability

\begin{tabular}{lrr}
\hline Parameter & $\begin{array}{r}\text { Right ascension } \\
{[\text { mas } / \mathrm{a}]}\end{array}$ & $\begin{array}{r}\text { Declination } \\
{[\text { mas } / \mathrm{a}]}\end{array}$ \\
\hline$\sigma_{\mathrm{c}}$ & 2.2 & 2.1 \\
$\sigma_{\mathrm{f}}$ & 13.3 & 5.6 \\
$X_{\mathrm{c}}, Y_{\mathrm{c}}$ & 0.0 & +1.0 \\
$X_{\mathrm{f}}, Y_{\mathrm{f}}$ & 0.2 & -3.8 \\
\hline
\end{tabular}

$\sigma_{\mathrm{c}}$ : proper motion dispersion of cluster stars $\sigma_{\mathrm{f}}$ : proper motion dispersion of field stars $X_{\mathrm{c}}, Y_{\mathrm{c}}$ : centre of the cluster star distribution $X_{\mathrm{f}}, Y_{\mathrm{f}}$ : centre of the field star distribution

algorithm was used. The reduction model included polynomials up to second order of the rectangular coordinates. The final catalogue contains positions and proper motions of 2220 stars with a median accuracy of 1.6 mas/a for the proper motions. Table 5 lists the proper motion differences of our catalogue with respect to the catalogues of Francic (1989) and the PPM. Since our proper motions do not refer to the PPM system or the same system as used in Francic (1989), only the rms. of our comparison is of importance. We note that the differences with respect to the PPM system are in good agreement with the internal errors of the PPM proper motions. Due to the higher precision of the catalogue of Francic (1989) we may assume that the main effect of the deviations to the Francic catalogue is caused by the uncertainty of our proper motions. The deviations are in fair agreement with the mean error of $1.7 \mathrm{mas} / \mathrm{a}$ for one proper motion component of our catalogue. The slightly higher value may be caused either by the different systems of proper motions, or by still undetected systematic effects of the positions at the border of the Carte du Ciel plates. Table 7 (accessible in electronic form) lists the positions and proper motions of 2220 stars in the field of NGC 1647.

From our catalogue we have selected all stars within a radius of $30^{\prime}$ around the cluster centre. The latter was determined from visual inspection of Palomar plates and was very near to the PPM star No. $120214\left(\alpha_{2000}=04 \mathrm{~h} 45 \mathrm{~m} 42 \mathrm{~s}\right.$, $\delta_{2000}=+19^{\circ} 07^{\prime} 07^{\prime \prime}$. 375 stars were selected. Using the method of Sanders (1971) we have fitted a bivariate Gaussian curve to the proper motion distribution and determined membership probabilities of the stars. Table 6 gives the values of the centre and the rms of the cluster members and the field stars. The corresponding vector point plot diagram is given in Fig. 3. The membership designations of the single stars are in good agreement with the data of Francic (1989). Assuming a membership of $50 \%$ for a cluster member, we found only in eight cases differences. Because the accuracy of our data is inferior to that of Francic (1989) we would expect that some non-member stars according to Francic appear as members according to our data. However, unexplanable discordance in the membership designations remained only for three stars.

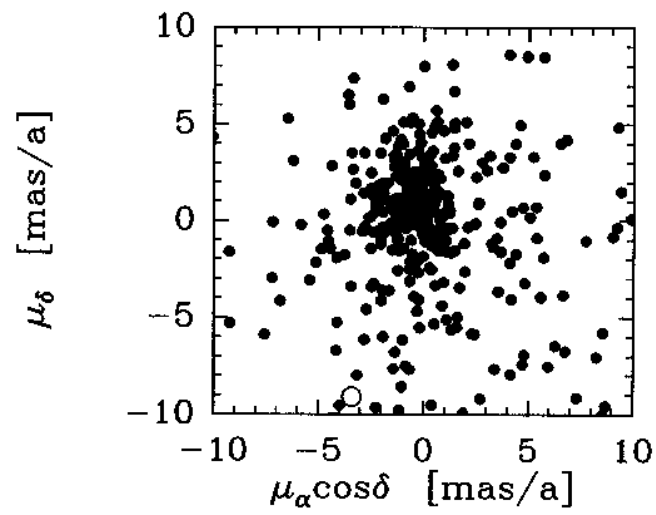

Fig. 3. The vector point plot diagram of stars with distance $R$ smaller than 30 arcmin from the cluster centre. The open circle indicates the proper motion of the Cepheid SZ Tau (see Sect. 7)

\section{On the membership of SZ Tau}

Cepheids as members of open clusters are interesting for the calibration of the period-luminosity function of galactic Cepheids. SZ Tau is a Cepheid, which lies about 2 degrees from the centre of NGC 1647. Turner (1992) discusses the membership of the Cepheid SZ Tau to NGC 1647. Due to coincidence in age, radial velocity and proper motions he concluded that SZ Tau is a member of the cluster. However, Turner (1992) uses only the proper motions of AGK3 stars. SZ Tau (BD +18 661, PPM 120081 ) is listed in the PPM with a proper motion of $\mu_{\alpha} \cos \delta=-7.1 \mathrm{mas} / \mathrm{a}$ and $\mu_{\delta}=-10.0 \mathrm{mas} / \mathrm{a}$. Assuming the mean difference of our proper motion system and the PPM system from the last chapter, SZ Tau should have a proper motion in our system of $\mu_{\alpha} \cos \delta=-3.4$ mas/a and $\mu_{\delta}=-9.1 \mathrm{mas} / \mathrm{a}$, which would correspond to a membership probability of below $10 \%$. We confirm therefore our earlier conclusion (Geffert et al. 1990), that astrometric evidence for SZ Tau beeing a member of NGC 1647 is not valid. Nevertheless, we cannot rule out that the proper motion system of the PPM may vary over 2 degree, and that the differences in proper motions are due to such differences. We expect a final solution to this problem when the results of the HIPPARCOS mission become available.

\section{Conclusions}

We have shown that the accuracy of Carte du Ciel plates is sufficient to use these plates as first epoch plates to extend the HIPPARCOS proper motion system to stars with magnitude down to $V=14$. Using a multithreshold technique of determining positions from triple images of 
Carte du Ciel plates lead to accuracies of 200 mas and better for each exposure even at the border of the plates. For the multithreshold procedure it seems to be more useful to take only the position of the highest threshold, in which the object could be detected (method A) than the mean of the positions from all thresholds.

Together with second epoch material we have derived proper motions for more than 2000 stars. The accuracy of the proper motions is of the order of 2 mas/a, which corresponds very well to the HIPPARCOS accuracy of proper motions. The accuracy of the proper motions seems, at least for some open clusters, to be sufficient to separate cluster and field stars. In our study we have obtained very similar membership probabilities as the study of Francic (1989). Moreover, we found from our proper motion study evidence that SZ Tau is not a member of NGC 1647.

Acknowledgements. The financial support of PROCOPE is gratefully acknowledged. We thank also for genereous allocation of measuring time in Paris and Münster. M.G. thanks the Observatoire de Bordeaux, where a major part of this investigation was done during a stay in April 1995, for its hospitality. We are also indebted to K.S. de Boer, A. Ortiz-Gil and M. Odenkirchen (Bonn) for helpful discussions. It is a pleasure to thank the referee B.F. Jones (Lick Observatory) for helpful comments.

\section{References}

Berger J., Cordoni J.P., Fringant A.M., Guibert J., Moreau O., Reboul H., Vanderriest C., 1991, A\&AS 87, 389

Bonnefond P., 1991, DEA thesis, Observatoire de Paris

Brosche P., Geffert M., 1988, Astrometry of Carte du Ciel plates. In: Debarbat S., Eddy J.A., Eichhorn H.K., Upgren A.R. (eds.) Mapping the Sky, IAU Symp. 133. Kluwer Dordrecht, p. 403

Brosche P., Wildermann E., Geffert M., 1989, A\&A 211, 239

Dick W.R., Tucholke H.-J., Brosche P., Galas R., Geffert M., Guibert J., 1993, A\&A 279, 267

Francic S., 1989, AJ 98, 888

Geffert M., Guibert J., Ducourant C., 1990, First proper motion study of the open cluster NGC 1647. In: Capitaine N. and Débarbat S. (eds.) Journées 1990: Systèmes de Référence spatio temporels, Colloque André Danjon, Observatoire de Paris 1990, p. 215

Geffert M., Kümmel M., Schmidt H., 1995, A\&AS 112, 229

Guibert J., Soubiran C., Geffert M., 1990, Photographic astrometry with MAMA. In: Capitaine N. and Débarbat S. (eds.) Journées 1990: Systèmes de Référence spatio temporels, Colloque André Danjon, Observatoire de Paris 1990, p. 211

Lattanzi, M.G., Massone G., Munari U., 1991, AJ 102, 177

Ortiz-Gil A., Brosche P., Hiesgen M., 1995, Astr. Ges. Abstr. Ser. 11, 92

Sanders W.L., 1971, A\&A 14, 226

Turner D.G., 1992, AJ 104, 1865 